\title{
HODGKIN'S DISEASE IN CHILDREN
}

\author{
BY
}

D. J. M. MCCAUSLAND, M.D.

This disease was first described in 1832 by Hodgkin. It is a subacute or chronic illness characterized by painless and progressive enlargement of lymph nodes, involvement of other organs in a widespread pathological process, sometimes accompanied by fever, and resulting in anaemia, cachexia and finally death. The disease occurs more often in males than females in the ratio of two to one, and more often in adults than children. In a series of 173 cases in Baltimore only 2.5 per cent. were in children.

The etiology of the disease is still undecided, some believing it to be an infectious granuloma and others that it is a neoplasm. The coincidence of tuberculosis and Hodgkin's disease in the same patient has been confusing. Tubercle bacilli cannot be isolated from pure cases of Hodgkin's disease, and so far it has not been transmitted to lower animals.

Pathologically there is a hyperplasia of the lymphoid tissues everywhere, the superficial and deep nodes, and the lymphoid tissue of bones, organs and nervous system. Histologically there is proliferation of the reticulo-endothelial cells, eosinophils and lymphoid cells. Certain of the reticulo-endothelial cells assume the giant multinucleated form known as Dorothy Reed cells. In the later stages fibrosis may replace most of the cells. The glands on examination vary in consistency from soft to elastic or hard, depending on the stage of the disease. They are discrete and not attached to skin. A single group may be involved at the onset, but the involvement soon spreads.

Blood findings are not diagnostic. A progressive secondary anaemia is characteristic. Usually there is a normal total leucocyte count or a slight leucopenia. In more advanced cases the total white count is increased with a polymorph leucocytosis, a relative increase of monocytes and decrease in lymphocytes.

The first symptom is usually a painless swelling of one or more groups of superficial nodes. The areas most frequently involved are, in order, the cervical, supraclavicular, axillary and inguinal. A characteristic of Hodgkin's disease in its early stages is that unilateral lymph areas are attacked with greater frequency than symmetrical areas. In other forms of lymphoblastoma, such as lymphosarcoma and leukaemia, the involved nodes are more apt to be symmetrically enlarged.

Often an acute upper respiratory tract infection, a dental abscess, or one of the acute exanthemata of childhood precedes the onset. In some cases the 
first glands involved are mediastinal or mesenteric, causing cough, dyspnoea, gastro-intestinal symptoms or a sense of heaviness in the abdomen due to an enlarged spleen. In others weakness, loss of weight, pallor, or unexplained fever, may be the first symptoms.

In a series of nine cases in children reported by Limper (1939), the initial symptoms were enlarged superficial glands in three; cough, with or without fever, in four; herpes zoster in one; and fever, pallor and haemorrhages in one. In the past ten years there have been thirteen cases of Hodgkin's disease admitted to this hospital. The initial symptoms were: fever in five cases; loss of strength in five cases; pallor in four cases; cough in two cases. Only three of the thirteen cases had the Pel-Ebstein type of fever. Limper reports only two of his nine cases in children having this symptom.

Splenomegaly and hepatomegaly are infrequent in early cases, but the spleen is enlarged in from 60 per cent. to 70 per cent. of advanced cases, and the liver in about 35 per cent. Both nodular and diffuse infiltration of bone marrow are common, and most frequently are seen in the vertebrae in children, and less often in the sternum and femur. The $x$-ray picture is that of osteolysis, resembling metastatic carcinoma. Cough and dyspnoea are the most frequent symptoms of mediastinal gland involvement, which does not occur without palpable cervical, supraclavicular, and axillary adenopathy. There is nothing pathognomonic in the $x$-ray picture of mediastinal Hodgkin's disease, which shows thickening of the hilar areas going on to a widening of the mediastinum, and invasion of adjacent lung fields. Pleural effusion is rare in children with mediastinal Hodgkin's disease.

Some type of cutaneous manifestation occurs in about 30 per cent. of cases, and includes simple pruritus, ulcerated papules similar to scabies, herpes zoster, and generalized exfoliative dermatitis. Pigmentation is frequently seen.

Of the thirteen cases admitted to the Hospital for Sick Children, Toronto, in the past ten years, eleven have been boys. The youngest was four years old and the oldest was twelve. The average age was eight years. The average duration of symptoms before death was nineteen months, the shortest period was three months, and the longest four and a half years. Eleven of these children had enlarged cervical glands, and one had enlarged axillary glands, on admission. Blood findings were not characteristic, but showed a varying degree of secondary anaemia, and decrease in lymphocytes.

The clinical course of Hodgkin's disease may be divided into three periods:

(1) The latent period, which is usually protracted, lasting from several months to several years, and usually causing no subjective symptoms. During this period involvement of cervical nodes occurs in 50 to 75 per cent. of cases, axillary, retroperitoneal, inguinal and mediastinal glands being involved less frequently. However, Ewing believes that though enlargement of cervical glands first attracts attention, primary enlargement of abdominal nodes, alone or combined with thoracic gland enlargement, occurs ten times more frequently than cervical primary enlargements, which are really extensions from an internal lesion.

(2) The period of progress and generalization. The feature of this period 
lies in the appearance of pressure symptoms. In the neck, tracheal compression causes dyspnoea, which increases till the patient dies of strangulation; hoarseness of aphasia may result from pressure on the recurrent laryngeal nerve; pressure on the brachial plexus by masses above and behind the clavicle produces pain, trophic changes, and paraesthesia. In the axilla glandular enlargement may cause limitation of arm movement, and symptoms of pressure on nerves, veins and lymphatics. In the mediastinum, pressure on the trachea causes cough, dyspnoea, and the development of emphysema. Pleural transudates when they occur are usually serous and occasionally bloody. Direct pressure on the heart may cause cardiac embarrassment and displacement to the left. Jaundice may occur from compression of bile ducts by abdominal nodes. Oedema of the lower extremities may follow pressure on the vena cava. Cases with marked splenomegaly experience the characteristic heaviness and dragging sensation. Gastro-intestinal Hodgkin's disease is rare in children and usually simulates ulcerative colitis or may cause intestinal obstruction.

Fever is almost invariably present and often of the Pel-Ebstein type. Fever is not pronounced in the more chronic forms and some cases may run an entirely afebrile course. Weakness and prostration may be prominent, but more often the child is able to pursue his activities for months or even years. No dysfunction of the kidneys occurs except for an occasional mild albuminuria.

(3) As the disease progresses there is an exaggeration of the constitutional symptoms and the period of cachexia occurs with profound anaemia, hypotension, cardiac dilatation, and often anasarca. Those who are not strangled die of anaemia, or myocardial degeneration. Many die of intercurrent infections.

The diagnosis should be made as early as possible and should rest on biopsy, but the histological picture of hyperplastic tuberculous lymphadenitis, aleukaemic reticulosis, and retothelial sarcoma may be indistinguishable from Hodgkin's disease. The Gordon test, which was to have been a specific diagnostic test for Hodgkin's disease, has been found to depend solely on the presence of eosinophils in the material injected and has nothing to do with the disease itself. Eosinophils are present in 70 per cent. of glands of Hodg'in's disease, and it is in the same percentage of cases that the Gordon test is positive.

The most common complication in Hodgkin's disease is tuberculosis, which occurred in fourteen of 150 cases of Hodgkin's disease in one series. Emphysema, bronchiectasis, and pyogenic infections of lungs may complicate mediastinal involvement. Septicaemia, usually streptococcal, and endocarditis occur occasionally.

Treatment consists in irradiation of the enlarged glands, either with radium or with x-ray. Arsenic is useful, especially in relieving pruritus. Treatment of anaemia is indicated. Surgery is advisable in some cases for the relief of pressure symptoms. Malignant granulomatosis, by reason of the lymphoid character of its lesions, is exceptionally radio-sensitive. The effect of $x$-ray therapy on the granulomatous tissues is destructive, and is followed by fibrosis in excess of that usually observed in untreated cases. The aim of treatment is to obtain as long remissions as possible by methodical destruction of all foci, deep 
and superficial. Simple daily irradiation, using the rotation plan, for about five weeks, until sufficient total dose has been given, is apparently the method of choice. The average duration of survival is prolonged, and may be doubled or tripled.

The prognosis in untreated cases is hopeless. In the acute cases treatment offers little. It is best in those cases with enlargement of superficial glands, and is not as good in those cases with involvement of mesenteric or mediastinal glands. Burnam, in 1926, reported a series of 173 cases, of which twenty-eight were regarded as cured after periods of one to ten years. Since then Holt (1933) reports that Burnam has some patients who have had no recurrence after as long as fifteen years.

The average duration in Corbeille's series of children (1928) was $22 \frac{1}{2}$ months. One lived $5 \frac{1}{2}$ years.

\section{Summary}

Hodgkin's disease occurs with comparative rarity in children. Diagnosis depends on biopsy, and the blood picture is not diagnostic. It has been possible to follow seven of the thirteen cases admitted to this hospital in the past ten years. Six of these are dead after an average of nineteen months from onset of symptoms. The seventh is still under treatment. Treatment consists in $\mathrm{x}$-ray therapy, the aim being to obtain as long remissions as possible. Although cures have been reported by some authors, none has occurred in cases treated in the Hospital for Sick Children, Toronto, in the past ten years.

\section{REFERENCES}

Corbeille, C. (1928). Minn. Med., 11, 678.

Burnam, C. F. (1926). J. Amer. med. Ass., 87, 1445.

Holt, L. E. (1933). Diseases of Infancy and Childhood, tenth edition, New York. 582.

Limper, M. A. (1939). Kentucky med. J., 37, 97. 